\title{
Tapping-mode tuning-fork near-field scanning optical microscopy of low power semiconductor lasers
}

\author{
N. H. LU*, W. C. LIN† \& D. P. TSAI† \\ *Department of Electronic Engineering, Sze-hai Institute of Technology and Commerce, Tuchen \\ Taipei County, 236 Taiwan, Republic of China \\ †Department of Physics, National Taiwan University, No.1, Sec. 4, Roosevelt Road, Taipei, 10617 \\ Taiwan, Republic of China
}

Key words. Evanescent waves, near-field scanning optical microscopy, semiconductor laser, tapping-mode.

\begin{abstract}
Summary
The newly developed inverted tapping-mode tuning-fork near-field scanning optical microscopy (TMTF-NSOM) is used to study the local near-field optical properties of strained AlGaInP/ $/ \mathrm{Ga}_{0.4} \mathrm{In}_{0.6} \mathrm{P}$ low power visible multiquantum-well laser diodes. In contrast to shear-force mode NSOM, TMTFNSOM provides the function to acquire the evanescent wave intensity ratio $|I(2 \omega)| /|I(\omega)|$ image, from which the evanescent wave decay coefficient $q$ can be evaluated for a known tapping amplitude. Moreover, we probe the near-field stimulated emission spectrum, which gives the free-space laser light wavelength $\lambda_{o}$ and the index of refraction $n_{\mathrm{r}}$ of the laser diode resonant cavity. Once $q, \lambda_{\circ}$, and $n_{\mathrm{r}}$ are all measured, we can determine the angle of incidence $\theta_{\mathrm{o}}$ of the dominant totally internally reflected waves incident on the front mirror facet of the resonator. Determination of such an angle is very important in modelling the stability of the laser diode resonator.
\end{abstract}

\section{Introduction}

Semiconductor lasers have become indispensable commercial components to our everyday life. For example, they are widely used in an increasing number of applications ranging from the light sources for optical information processing systems such as bar-code scanning, high-speed laser printing, and optical read/write recording to the transmitters in optical fibre telecommunications in recent years. Accordingly, characterization and further understanding of the performance and reliability of various semiconductor laser structures turn out to be important tasks. Because of the superior spatial resolution of near-field

Correspondence to: Din Ping Tsai. Tel: +88622362 6937, ext. 336; fax: +886 22363 9984; e-mail: dptsai@phys.ntu.edu.tw scanning optical microscopy (NSOM) and the ease of implementation of local spectroscopy, lately NSOM has played a key role in optical imaging and spectroscopic study in the diagnostics of semiconductor lasers (Hörsch et al., 1996; Lienau et al. 1997; Ben-Ami et al., 1998; Kim et al., 2000).

Recently Lu et al. (1999) applied both collection and excitation modes of NSOM to study the optical properties of low power strained $\mathrm{AlGaInP} / \mathrm{Ga}_{0.4} \mathrm{In}_{0.6} \mathrm{P}$ visible multiquantumwell laser diodes (MQWLDs). The results show that both the near-field optical beam induced current and the near-field electroluminescence can be studied in the same region on a nanometer-sized length scale, which demonstrates the effective advantages of NSOM - that different operation modes of NSOM can serve as complementary local characterization techniques to one another. Furthermore, the defect location and the energy range of defect level can be resolved by the excitation mode of NSOM using pump sources of different wavelengths.

In this paper, the newly developed novel method, tappingmode tuning-fork near-field scanning optical microscopy (TMTF-NSOM) (Tsai \& Lu, 1998), is used to study the local near-field optical properties of low power visible MQWLDs. As has been demonstrated by Tsai et al. (1999), TMTFNSOM has the ability to perform evanescent wave intensity ratio imaging. Thus we can determine the decay coefficient of the evanescent fields due to the totally internally reflected waves incident on the front mirror facet of the laser diode (LD) resonant cavity, on condition that the tapping amplitude of the fibre tip is small and known.

\section{Experimental}

The inverted TMTF-NSOM was used to probe the near-field optical signals on the emission edge of the $\mathrm{LD}$ by collection mode. A schematic of the experimental set-up is shown in Fig. 1. For imaging acquisition, light coupled to the tapered 


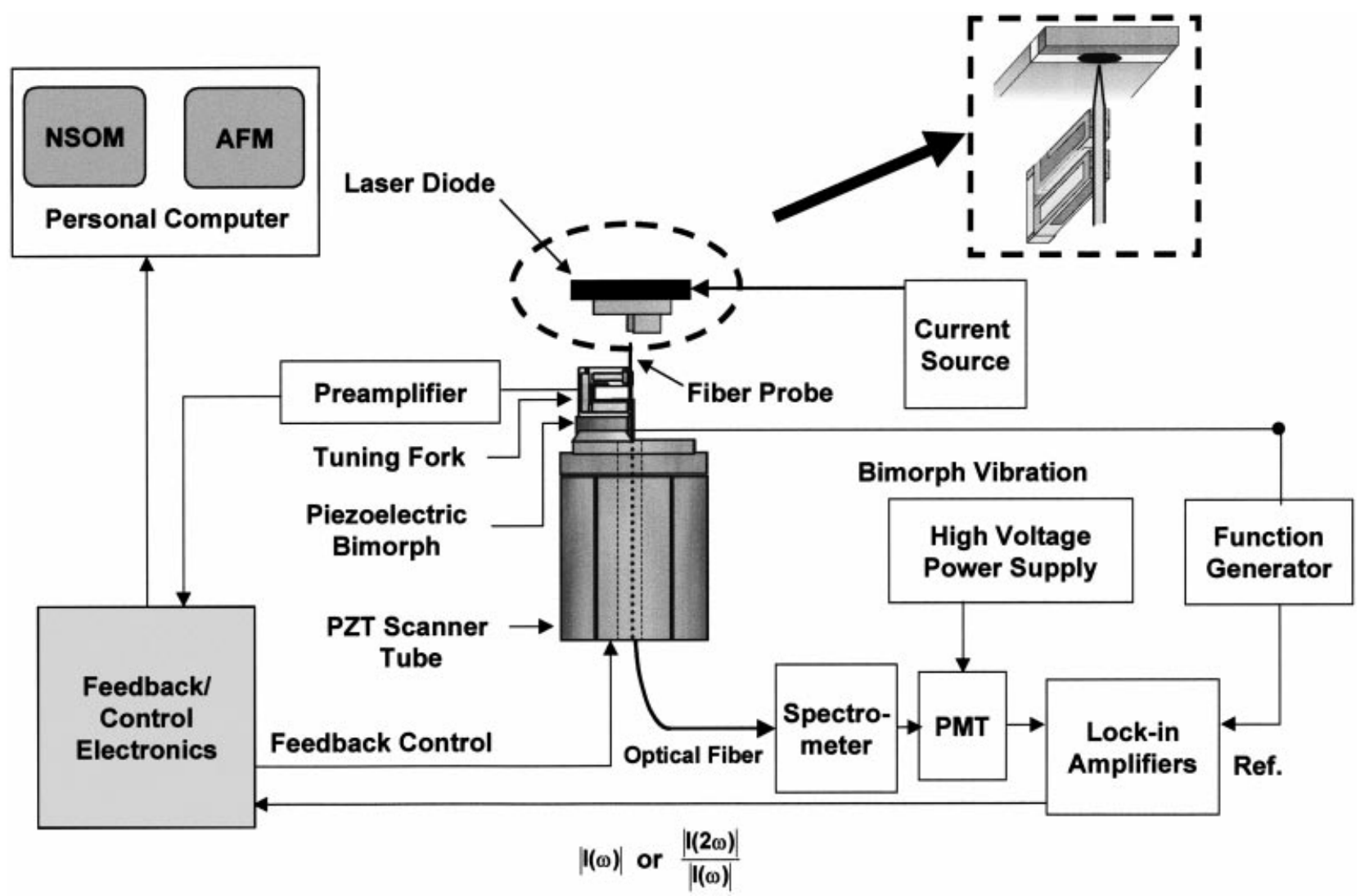

Fig. 1. A schematic diagram of the experimental set-up for measuring the near-field intensity ratio image on the emission facet of a laser diode. An inverted TMTF-NSOM system with two external lock-in amplifiers is used to simultaneously provide images of both the topography and the optical intensity signals of $|I(\omega)|,|I(2 \omega)|$, or their intensity ratio $|I(2 \omega)| /|I(\omega)|$.

fibre tip engaged in the near-field region was detected by a photomultiplier tube, the output signal of which was fed to two external lock-in amplifiers. The lock-in amplifiers acquired signals at the tapping frequency $\omega$ and its second harmonic counterpart $2 \omega$, and provided their intensity ratio $|I(2 \omega)| /|I(\omega)|$. The distance regulation between the sample surface and the cusp of the fibre probe was realized by another internal lock-in amplifier of a commercial electronic control unit of an atomic force microscope (AFM), which has been described elsewhere (Tsai \& Lu, 1998; Tsai et al., 1999). The feedback control of the probe height is a non-optical method and has all the advantages afforded by the tapping-mode AFM scheme, including high spatial resolution, high sensitivity, and excellent stability. Images of the topographic and near-field optical signals are acquired simultaneously. For spectroscopic characterization, the light collected by the tapered fibre probe was delivered to a $0.3-\mathrm{m}$ optical spectrometer fitted with a liquid nitrogen cooled multichannel charge-coupled device (CCD). The spectral resolution of our system is $0.1 \mathrm{~nm}$. The epitaxial structure of the LD is similar to that reported elsewhere (Lu et al., 1999), except that the quantum well thickness is $5 \mathrm{~nm}$ and the resonant cavity length $L, 400 \mu \mathrm{m}$.

\section{Results and discussion}

In contrast to shear-force mode NSOM, which can obtain the near-field emission intensity signal, TMTF-NSOM provides the function to acquire the evanescent wave intensity ratio $|I(2 \omega)| /|I(\omega)|$ image. The near-field optical intensity $I$ emanating from an LD emission surface can be expressed as $I=I_{\mathrm{p}}+I_{\mathrm{o}} e^{-2 q z}$, where $I_{\mathrm{p}}$ represents the constant propagating term, $I_{\mathrm{o}}$ denotes the evanescent wave intensity at the surface of the LD emission edge, $z$ is the probe height above the LD emission surface, and $q$, the decay coefficient. Given that the index of refraction of the semiconductor laser resonant cavity is 3.0, the totalinternal-reflection angle of the resonator/air interface is $19.5^{\circ}\left(=\sin ^{-1} 1 / 3\right)$. The evanescent component is due chiefly to the light waves propagating within the resonator when they strike the front mirror facet at an angle of incidence $\theta_{0}$ beyond the total-internal-reflection angle, which is a condition easily satisfied. The decay coefficient is derived as

$$
q=\left(2 \pi / \lambda_{0}\right)\left(n_{\mathrm{r}}^{2} \sin ^{2} \theta_{\mathrm{o}}-1\right)^{1 / 2}
$$

where $n_{\mathrm{r}}$ is the index of refraction of the LD resonant cavity 
and $\lambda_{\mathrm{o}}$ is the free-space light wavelength (van Hulst et al. 1992). Based upon the above argument, the actual angle of incidence of the dominant totally internally reflected waves incident on the output coupler can be experimentally determined if $\lambda_{\mathrm{o}}, n_{\mathrm{r}}$ and $q$ are all measured. This will be demonstrated below.

Instead of working in the constant height (constant $z$ ) mode in conventional near-field microscopes, $z$ is modulated in TMTF-NSOM: $z=z_{\mathrm{o}}+A \cos \omega t$, in which $A$ and $z_{0}$ represent the tapping amplitude of the probe and the closest distance between tip and sample surface, respectively (Tsai et al., 1999). For a small tapping amplitude (i.e. $q A<1$ ), the evanescent wave intensity ratio of $|I(2 \omega)|$ to $|I(\omega)|$ can be approximated as $|I(2 \omega)| /|I(\omega)| \cong\left[q A+(1 / 3) q^{3} A^{3}\right] /$ $\left(2+q^{2} A^{2}\right)$. Therefore, measurement of $|I(2 \omega)| /|I(\omega)|$ will directly give the value of $q$ if the tapping amplitude is known.

Plotted in Fig. 2(a) and (b) are the $5 \mu \mathrm{m} \times 5 \mu \mathrm{m} \mathrm{AFM}$ topography and evanescent wave intensity ratio $|I(2 \omega)| /$ $|I(\omega)|$ image of the emitted light at the front mirror facet of a strained AlGaInP/GaInP MQWLD operating with injection current just above its threshold. As is shown, the intensity ratio image shows one ellipse-like spot just below the $p$ electrode in the positive-intrinsic-negative junction. Also displayed in the inset is the intensity ratio linecut along the central line perpendicular to the MQW epitaxial layers. Obviously the profile exhibits a plateau structure with a smooth fall-off on the edge. Actually the intensity ratio image displays a rather uniform output distribution over the entire brighter contrast region. The full width at half maximum (FWHM) of the cross-sectional evanescent wave intensity ratio profile in the direction normal to the epitaxial layers is $762 \mathrm{~nm}$ and that in the direction parallel to the epitaxial layers is $3.0 \mu \mathrm{m}$.

The intensity ratio signals give the value of $q$ if the tapping amplitude is known (Tsai et al., 1999). The tapping amplitude is estimated to be $65 \mathrm{~nm}$ from an interferometric measurement. Since $|I(2 \omega)| /|I(\omega)|=0.33$, the decay coefficient $q$ is determined to be $0.011 \mathrm{~nm}^{-1}$. The evanescent fields emerge from $\mathrm{LD}$ resonator to air with an exponentially decreasing density of energy transferred in the $z$ direction. Obviously the evanescent wave intensity decay length, defined as $d_{\mathrm{p}}=1 / 2 q$, is calculated to be $45 \mathrm{~nm}$.

Figure 3 shows the near-field stimulated emission spectrum of the LD when the tip is positioned at the centre of the active region. The injection current is the same as that operated in Fig. 2. As can be seen, the emission spectrum exhibits multiple peak structures around their centre wavelength, $654.49 \mathrm{~nm}$, and the free spectral range is $\Delta \lambda_{\mathrm{FSR}}=0.17 \mathrm{~nm}$. Since the wavelength dependence of $n_{\mathrm{r}}$ is negligible in our case (Adachi et al., 1994), the free spectral range can be given by $\Delta \lambda_{\mathrm{FSR}}=\lambda_{\mathrm{o}}^{2} / 2 n_{\mathrm{r}} L$, where $\lambda_{\mathrm{o}}$ is chosen to be the centre wavelength in vacuum in our calculation. Therefore, the refractive index of the $\mathrm{LD}$ structure is readily calculated as $n_{\mathrm{r}}=3.15$. Substituting $n_{\mathrm{r}}=3.15, \lambda_{\mathrm{o}}=654.49 \mathrm{~nm}$, and the previously determined $q=0.011 \mathrm{~nm}^{-1}$ into Eq. (1), we can obtain $\theta_{\mathrm{o}}=29^{\circ}$. The result shows that the evanescent waves are due chiefly to the inside-cavity propagating waves incident on the front mirror facet at an angle of $29^{\circ}$. Determination
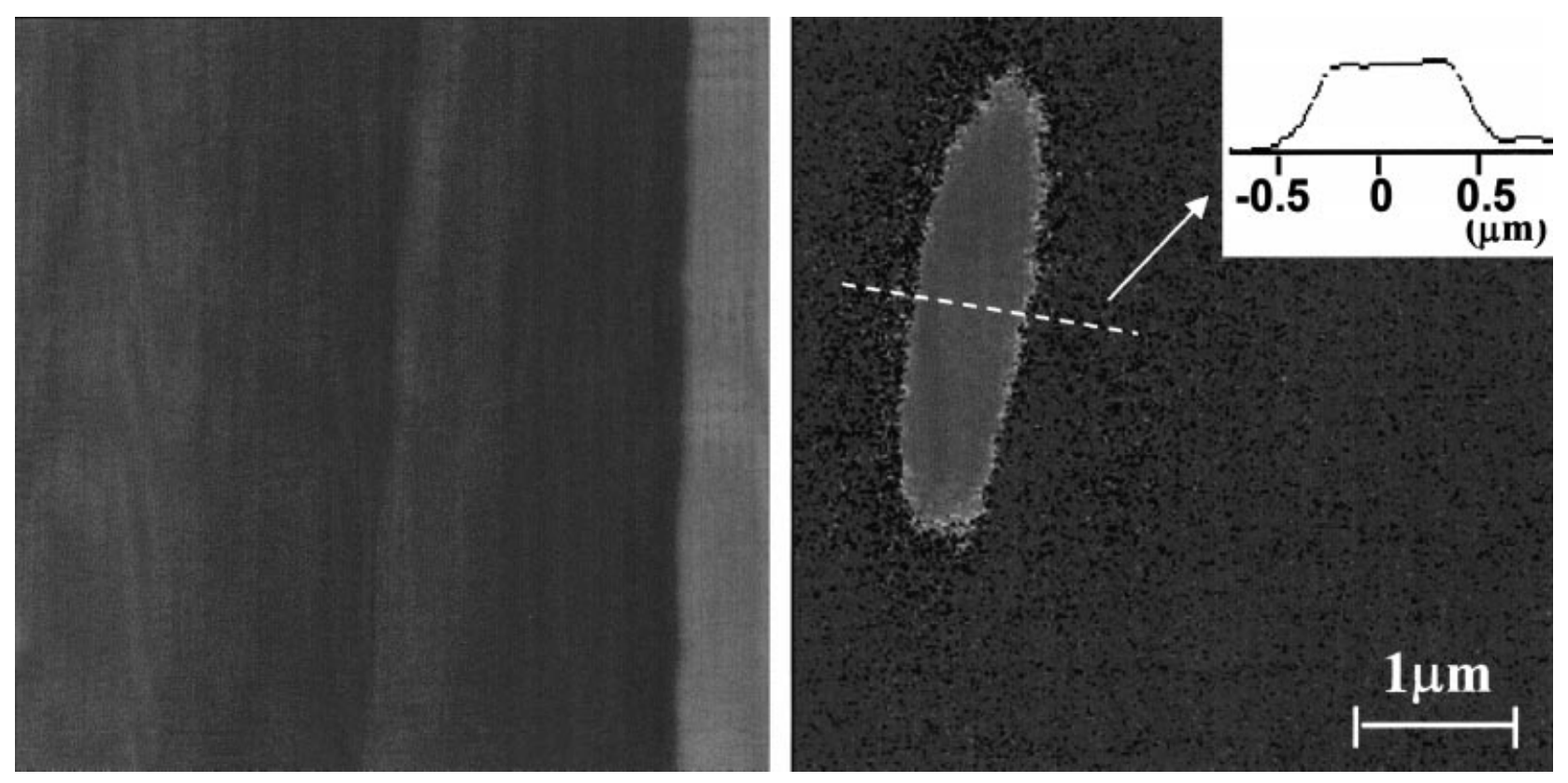

Fig. 2. $5 \mu \mathrm{m} \times 5 \mu \mathrm{m}$ scanned NSOM images of (a) AFM topography and (b) evanescent wave intensity ratio $|I(2 \omega)| /|I(\omega)|$ on the emission facet of a strained $\mathrm{AlGaInP} / \mathrm{Ga}_{0.4} \mathrm{In}_{0.6} \mathrm{P}$ low power visible $\mathrm{MQWLD}$ operating just above the threshold current. Also shown in the inset is the intensity ratio linecut along the central line perpendicular to the MQW epitaxial layers. 


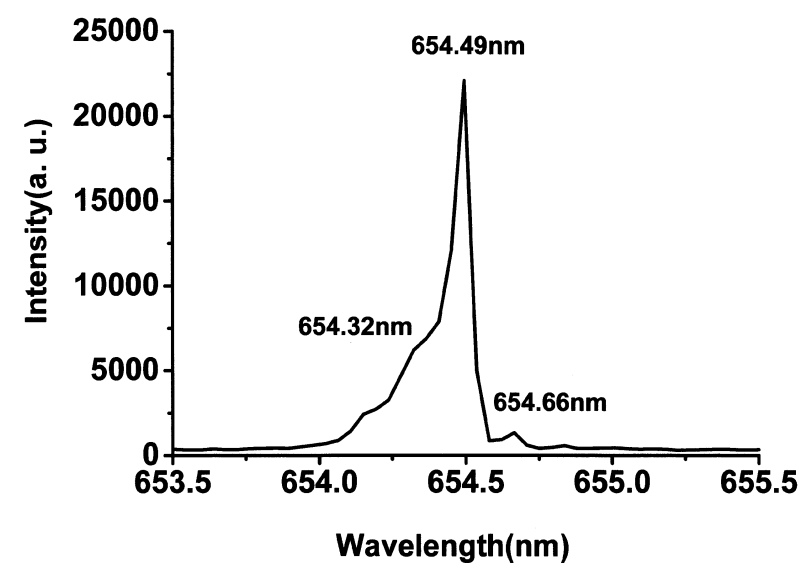

Fig. 3. Near-field stimulated emission spectrum of the LD when the tip is positioned at the centre of the active region. The spectral output exhibits several peak structures around their centre wavelength $(654.49 \mathrm{~nm})$ with a constant peak separation of $0.17 \mathrm{~nm}$

of such an angle is very important in modelling the stability of the laser diode resonator.

\section{Conclusions}

As has been pointed out by van Hulst et al. (1992), there exists problems in isolating the evanescent field component when lateral-force mode evanescent field optical microscope (or photon scanning tunneling microscope) is applied to probe the evanescent wave generated by total internal reflection at a substrate surface, on which roughness induced field mixing of the radiative waves with the evanescent ones is inevitable. They also suggest that the $z-$ modulation technique should be one of the possible solutions. Here we have demonstrated how TMTF-NSOM can be used to measure the evanescent wave decay coefficient of the light emanating from the $\mathrm{LD}$ facet if the tapping amplitude of the fibre probe is small and known. Furthermore, the light wavelength and the index of refraction of the resonant cavity are obtained from the near-field stimulated emission spectrum of the LD. In consequence, we can experimentally determine the angle of incidence of the dominant totally internally reflected beams incident on the front mirror facet of the LD.

\section{Acknowledgements}

The authors gratefully acknowledge the support of the National Science Council of the Republic of China. The work at Sze-hai Institute of Technology and Commerce was supported under Grant No. NSC 89-2112-M-237-001. The work at National Taiwan University was supported under Grant No. NSC 89-2112-M-002-083.

\section{References}

Adachi, S., Kato, H., Moki, A. \& Ohtsuka, K. (1994) Refractive index of $\left(\mathrm{Al}_{\mathrm{x}} \mathrm{Ga}_{1-\mathrm{x}}\right)_{0.5} \mathrm{In}_{0.5} \mathrm{P}$ quaternary alloys. J. Appl. Phys. 75, $478-480$.

Ben-Ami, U., Nagar, R., Ben-Ami, N et al. (1998) Near-field scanning optical microscopy studies of V-grooved quantum wire lasers. Appl. Phys. Lett. 73, 1619-1621.

Hörsch, I., Kusche, R., Marti, O., Weigl, B. \& Ebeling, K.J. (1996) Spectrally resolved near-field mode imaging of vertical cavity semiconductor lasers. J. Appl. Phys. 79, 3831-3834.

van Hulst, N.F., Segerink, F.B. \& Bölger, B. (1992) High resolution imaging of dielectric surfaces with an evanescent field optical microscope. Opt. Commun. 87, 212-218.

Kim, J., Boyd, J.T., Jackson, H.E. \& Choquette, K.D. (2000) Nearfield spectroscopy of selectively oxidized vertical cavity surface emitting lasers. Appl. Phys. Lett. 76, 526-528.

Lienau, Ch., Richter, A. \& Tomm, J.W. (1997) Near-field photocurrent spectroscopy: a novel technique for studying defects and aging in high-power semiconductor lasers. Appl. Phys. A, 64, $341-351$.

Lu, N.H., Tsai, D.P., Chang, C.S. \& Tsong, T.T. (1999) Optical characterization of visible multiquantum-well semiconductor lasers by collection/excitation modes of scanning near-field optical microscopy. Appl. Phys. Lett. 74, 2746-2748.

Tsai, D.P. \& Lu, Y.Y. (1998) Tapping-mode tuning fork force sensing for near-field scanning optical microscopy. Appl. Phys. Lett. 73, 2724-2726.

Tsai, D.P., Yang, C.W., Lo, S. \& Jackson, H.E. (1999) Imaging local index variations in an optical waveguide using a tapping-mode near-field scanning optical microscope. Appl. Phys. Lett. 75, 1039-1041. 